\title{
Frequency/Laplace domain methods for computing transient responses of fractional oscillators
}

\author{
Qianying Cao ${ }^{1}$, Sau-Lon James $\mathrm{Hu}^{2}$, Huajun $\mathrm{Li}^{3}$
}

\begin{abstract}
Although computing the transient response of fractional oscillators, characterized by secondorder differential equations with fractional derivatives for the damping term, to external loadings has been studied, most existing methodologies have dealt with cases with either restricted fractional orders or simple external loadings. In this paper, considering complicated irregular loadings acting on oscillators with any fractional order between 0 and 1, efficient frequency/Laplace domain methods for getting transient responses are developed. The proposed methods are based on pole-residue operations. In the frequency domain approach, "artificial" poles located along the imaginary axis of complex plane are designated. In the Laplace domain approach, the "true" poles are extracted through two phases: (1) a discrete impulse response function (IRF) is produced by taking the inverse Fourier transform of the corresponding frequency response function (FRF) that is readily obtained from the exact TF, and (2) a complex exponential signal decomposition method, i.e., the Prony-SS method, is invoked to extract the poles and residues. Once the poles/residues of the system are known, those of the response can be determined by simple pole-residue operations. Sequentially, the response time history is readily obtained. Two fractional oscillators with rational and irrational derivatives, respectively, subjected to sinusoidal and complicated earthquake loading are presented to illustrate the procedure and verify the correctness of the proposed method. The verification is conducted by comparing the results from both the Laplace and the frequency domain \footnotetext{
116024, PR China. E-mail: caoqianying@dlut.edu.cn

${ }^{2}$ Professor, Department of Ocean Engineering, University of Rhode Island, Narragansett, RI 02882-1197, USA. E-mail:jameshu@uri.edu

${ }^{3}$ Professor, Shandong Provincial Key Lab of Ocean Engineering, Ocean University of China, Qingdao 266100, PR China. E-mail:huajun@ouc.edu.cn
}

${ }^{1}$ Research fellow, State Key Lab of Coastal and Offshore Engineering, Dalian University of Technology, Dalian
\end{abstract}


approaches with those from the numerical Duhamel integral method.

Keywords: fractional oscillators, pole, residue, transient response, irregular loadings

\section{INTRODUCTION}

A second-order linear system with a fractional derivative damping term, which can capture the frequency-dependence of damping properties in viscoelastic materials, is called a fractional oscillator (Di Paola et al. 2012). On computing the responses of fractional oscillators, existing analytical solutions are limited to cases with specific fractional order $q$, such as $q=1 / 2$ and $q=1 / 3$, subjected to simple loadings, including impulse and step loading. In contrast, this paper develops efficient frequency/Laplace domain methods to compute the response of fractional oscillators with arbitrary fractional order to complicated irregular loadings.

Roughly, four distinct strategies in the literature have been pursued to compute the response of a fractional oscillator. As a common way to obtain the transient response of a linear system to any loading is through the Duhamel integral, which employs the impulse response function (IRF) of the system (Suarez and Shokooh 1995), a popular strategy has been attempting to obtain the fractional oscillator's IRF first (Gaul et al. 1989; Suarez and Shokooh 1995; Agrawal 1999; Agrawal 2001; Achar et al. 2002; Ye et al. 2003; Achar et al. 2004; Huang et al. 2010; Naber 2010; Pinnola 2016). While considering an oscillator with fractional order $q=1 / 2$, Gaul et al. (Gaul et al. 1989) computed the system's IRF by taking the inverse Fourier transform analytically from the system's frequency response function (FRF). Proceeding in the Laplace domain, Suarez and Shokooh (Suarez and Shokooh 1995) obtained the IRF of a fractional oscillator with $q=1 / 2$ or $q=1 / 3$, which involved solving a $4^{\text {th }}$-order polynomial for finding the poles of an oscillator with $q=1 / 2$, and a $6^{\text {th }}$-order with $q=1 / 3$. Using the generalized Mittag-Leffler functions, Achar et al. (Achar et al. 2002; Achar et al. 2004) expressed the IRF as a summation of infinity terms. Another common strategy for computing the fractional oscillator's response has been transforming the equation of motion into an alternative form so that it allowed to be solved by ordinary calculus (Bagley and Torvik 1983; Bagley and Calico 1985; Suarez and Shokooh 1997; Koh and Kelly 1990; Shokooh and Suárez 1999; Chang and Singh 2002; Yuan and Agrawal 2002; Cortés and 
Elejabarrieta 2007; Sheng et al. 2011; Mendiguren et al. 2012; Di Paola et al. 2012; Pinnola 2016; Zarraga et al. 2019). When $q$ is limited to a simple rational number, several papers (Bagley and Torvik 1983; Suarez and Shokooh 1997; Pinnola 2016) transformed the fractional differential equation into a set of equations in the so-called state variable domain where a set of decoupled fractional differential equations with the same fractional order was derived. The solution procedure has been operated in the Laplace domain, and each modal response must be obtained through an inverse Laplace transform. As this approach was often confronted with the difficulty of the inverse Laplace transform, the explicit response solutions of fractional oscillators to complicated loadings were difficult to obtain. Because of the difficulties of calculating inversion of Laplace transforms, Sheng et al. (Sheng et al. 2011) applied the numerical inverse Laplace transform algorithm in fractional calculus. This state variable analysis method not only requires $q$ be a rational number but also rapidly increases the number of state variables when the denominator of $q$ becomes large. The third strategy has been entirely numerical, which approximates the fractional derivative term at discrete times using central difference method (Koh and Kelly 1990; Shokooh and Suárez 1999) or Grünwald-Letnikov definition (Cortés and Elejabarrieta 2007; Mendiguren et al. 2012; Zarraga et al. 2019). The fourth strategy proposed in a few articles (Yuan and Agrawal 2002; Di Paola et al. 2012) involved the change of variable and integral approximation schemes to convert the fractional oscillator's equation of motion into a set of coupled linear equations with ordinary derivatives so that they could be computed by any time-domain numerical integration scheme.

This paper proposes novel frequency/Laplace domain methods based on pole-residue operations for computing the transient responses of fractional oscillators with arbitrary $q$ between 0 and 1 to complicated loadings. The key of the proposed method is to obtain the poles and residues of the system transfer function (TF) for fractional oscillators, then those of the response. Knowing the poles and residues of the response allows it to be expressed immediately in the time domain (Hu et al. 2016; Hu and Gao 2018). The proposed method includes the following steps: (1) obtaining the poles and residues of the TF for the fractional oscillator from the analytical FRF; (2) computing the poles and residues of the response from those of the TF and the excitation by simple pole- 
residue operations; and (3) expressing the response time history from the poles and residues of the response.

Two fractional oscillators with rational and irrational derivatives, respectively, subjected to sinusoidal and complicated earthquake loading are provided to illustrate the proposed method. In both examples, the response calculation will be carried out in both the frequency domain and the Laplace domain. The accuracy of the proposed method will be verified through a time domain approach that employs the numerical Duhamel integral.

\section{PRELIMINARIES}

\section{System functions}

The most essential background material to this study is the system functions, which are employed to characterize the relationship between the response (output) and the excitation (input) of a linear time-invariant system, including the IRF in the time domain, the FRF in the frequency domain, and the TF in the Laplace domain. Denoting the input and output signals associated with a linear system as $u(t)$ and $y(t)$, respectively, they are related in the time domain as

$$
y(t)=\int_{-\infty}^{\infty} h(t-\tau) u(\tau) d \tau
$$

where $h(t)$ is the system's IRF. In the frequency domain, one computes the steady-state response

$$
Y(\omega)=\mathcal{H}(\omega) U(\omega)
$$

where $\mathcal{H}(\omega)$ is system's FRF, which is the Fourier transform of $h(t)$

$$
\mathcal{H}(\omega) \equiv \mathcal{F}[h(t)]=\int_{-\infty}^{\infty} h(t) e^{-i \omega t} d t
$$

where $\mathcal{F}(\cdot)$ denotes the Fourier transform operator and $i=\sqrt{-1}$ is the imaginary number. Conversely, $h(t)$ is the inverse Fourier transform of $\mathcal{H}(\omega)$, namely

$$
h(t) \equiv \mathcal{F}^{-1}[\mathcal{H}(\omega)]=\frac{1}{2 \pi} \int_{-\infty}^{\infty} \mathcal{H}(\omega) e^{i \omega t} d \omega
$$


When the system is causal, i.e., $h(t)=0$ for $t \leq 0$, one writes the input-output relationship in the Laplace domain as

$$
\tilde{y}(s)=\tilde{h}(s) \tilde{u}(s)
$$

where $\tilde{h}(s)$ is system's TF, which is the Laplace transform of $h(t)$

$$
\tilde{h}(s) \equiv \mathcal{L}[h(t)]=\int_{0}^{\infty} h(t) e^{-s t} d t
$$

where $\mathcal{L}(\cdot)$ denotes the Laplace transform operator. Furthermore, $\mathcal{H}(\omega)$ can be also obtained directly from $\tilde{h}(s)$ by substituting $s$ with $i \omega$, that is

$$
\mathcal{H}(\omega)=\tilde{h}(i \omega)
$$

\section{Transfer functions of fractional oscillators}

For a fractional oscillator, the equation of motion is written as:

$$
m \ddot{x}(t)+c D_{0^{+}}^{q} x(t)+k x(t)=f(t)
$$

where $m, c$ and $k$ are the mass, damping and stiffness of the oscillator; $x(t), \dot{x}(t)$ and $\ddot{x}(t)$ are the displacement, velocity and acceleration of the structure response, respectively; $f(t)$ is the external loading; and $D_{0^{+}}^{q} x(t)$ for $0<q<1$ follows the definition of Caputo's fractional derivative (Caputo 1967; Matlob and Jamali 2019; Bagley and Torvik 1983)

$$
D_{0^{+}}^{q} x(t)=\frac{1}{\Gamma(1-q)} \int_{0}^{t}(t-\tau)^{-q} \dot{x}(\tau) d \tau
$$

in which $\Gamma(\cdot)$ is the gamma function. Since the Laplace transform of Eq. 9 is (Bagley and Torvik 1983)

$$
\mathcal{L}\left[D_{0^{+}}^{q} x(t)\right]=s^{q} \tilde{x}(s)
$$

taking the Laplace transform of Eq. 8 yields $\tilde{x}(s)=\tilde{h}(s) \tilde{f}(s)$ with the corresponding TF

$$
\tilde{h}(s)=\frac{1}{m s^{2}+c s^{q}+k}
$$

Substituting $s$ by $i \omega$ into Eq. 11 yields

$$
\mathcal{H}(\omega)=\tilde{h}(i \omega)=\frac{1}{-m \omega^{2}+c(i \omega)^{q}+k}
$$




\section{RESPONSE CALCULATION IN THE FREQUENCY DOMAIN}

The concept of the pole-residue method developed in Ref. (Hu et al. 2016) will be employed to compute $x(t)$. When the response computation is carried out in the frequency domain, many imaginary poles $i \hat{\omega}_{n}$ are assigned, where $\hat{\omega}_{n}=n \Delta \hat{\omega}$ and $n=$ integer. Referring to Eq. 12, and denoting $\mathcal{H}_{n}=\mathcal{H}\left(\hat{\omega}_{n}\right)$, one writes

$$
\mathcal{H}_{n}=\frac{1}{-m \hat{\omega}_{n}^{2}+c\left(i \hat{\omega}_{n}\right)^{q}+k}
$$

From Eq. 4, the system's IRF can be approximated as

$$
h(t) \approx \frac{1}{2 \pi} \sum_{n=-N_{n} / 2}^{N_{n} / 2} \mathcal{H}_{n} e^{i \hat{\omega}_{n} t} \Delta \hat{\omega}
$$

where $N_{n}$ must be sufficiently large so that $\mathcal{H}_{N_{n} / 2} \rightarrow 0$. Denote

$$
A_{n}=\mathcal{H}_{n} \Delta \hat{\omega} /(2 \pi)
$$

thus

$$
h(t) \approx \sum_{n=-N_{n} / 2}^{N_{n} / 2} A_{n} e^{i \hat{\omega}_{n} t}
$$

Noting that $A_{n} e^{i \hat{\omega}_{n} t}$ and $\frac{A_{n}}{s-i \hat{\omega}_{n}}$ form a Laplace transform pair (Kreyszig 2010) and performing the Laplace transform of Eq. 16 yields

$$
\tilde{h}(s) \approx \sum_{n=-N_{n} / 2}^{N_{n} / 2} \frac{A_{n}}{s-i \hat{\omega}_{n}}
$$

Now, $\tilde{h}(s)$ is in a pole-residue form with poles $i \hat{\omega}_{n}$ and corresponding residues $A_{n}$. Obviously, the inverse Laplace transform of Eq. 17 will go back to Eq. 16. An important fact drawn from Eqs. 16 and 17 is that once $i \hat{\omega}_{n}$ and $A_{n}$ are given, one can readily obtain $h(t)$ and $\tilde{h}(s)$.

For a periodic excitation $f(t)$ with period $T$, it can always be expressed as a complex Fourier series

$$
f(t)=\sum_{m=-N_{m} / 2}^{N_{m} / 2} C_{m} \exp \left(i \omega_{m} t\right)
$$


where $\omega_{m}=m \Delta \omega$ and $\Delta \omega=2 \pi / T$, and the corresponding complex Fourier coefficient

$$
C_{m}=\frac{1}{T} \int_{0}^{T} f(t) e^{-i \omega_{m} t} d t
$$

Because $f(t)$ is a real-valued signal, $C_{-m}$, the coefficient at $-\omega_{m}$, is equal to the complex conjugate of $C_{m}$. Theoretically, a Fourier series representation of $f(t)$ contains an infinite number of terms; however, in practice $f(t)$ can generally be approximated with sufficient accuracy by finite terms. For a digital signal sampled with a constant $\Delta t$, the fast Fourier transform (FFT) algorithm has been often invoked to compute $C_{m}$. From Eq. (18), the corresponding $\tilde{f}(s)$ is

$$
\tilde{f}(s)=\sum_{m=-N_{m} / 2}^{N_{m} / 2} \frac{C_{m}}{s-i \omega_{m}}
$$

This is a pole-residue form for a periodic function with poles $i \omega_{m}$ and residues $C_{m}$.

According to the operation in the Laplace domain $\tilde{x}(s)=\tilde{h}(s) \tilde{f}(s)$, using Eqs. 17 and 20, one writes

$$
\tilde{x}(s)=\left(\sum_{n=-N_{n} / 2}^{N_{n} / 2} \frac{A_{n}}{s-i \hat{\omega}_{n}}\right)\left(\sum_{m=-N_{m} / 2}^{N_{m} / 2} \frac{C_{m}}{s-i \omega_{m}}\right)
$$

First, assuming the chosen $\hat{\omega}_{n}$ and $\omega_{m}$ do not overlap, as the common denominator in Eq. 21 is $\left(s-i \hat{\omega}_{n}\right)\left(s-i \omega_{m}\right)$, the pole-residue form (partial-fraction form) of $\tilde{x}(s)$ is (Craig and Kurdila 2006)

$$
\tilde{x}(s)=\sum_{n=-N_{n} / 2}^{N_{n} / 2} \frac{Q_{n}}{s-i \hat{\omega}_{n}}+\sum_{m=-N_{m} / 2}^{N_{m} / 2} \frac{P_{m}}{s-i \omega_{m}}
$$

where

$$
Q_{n}=\sum_{m=-N_{m} / 2}^{N_{m} / 2} \frac{A_{n} C_{m}}{i\left(\hat{\omega}_{n}-\omega_{m}\right)}
$$

and

$$
P_{m}=\sum_{n=-N_{n} / 2}^{N_{n} / 2} \frac{A_{n} C_{m}}{i\left(\omega_{m}-\hat{\omega}_{n}\right)}
$$

Then, carrying out the inverse Laplace transform yields

$$
x(t)=\sum_{n=-N_{n} / 2}^{N_{n} / 2} Q_{n} \exp \left(i \hat{\omega}_{n} t\right)+\sum_{m=-N_{m} / 2}^{N_{m} / 2} P_{m} \exp \left(i \omega_{m} t\right)
$$


In Eq. 25, while the first summation term is the transient response related to the system poles $i \hat{\omega}_{n}$, the second summation term is the steady-state response related to the excitation frequencies $\omega_{m}$,

Second, if same poles are shared by $\tilde{h}(s)$ and $\tilde{f}(s)$, then second-order poles exist in Eq. 21 . When $\Delta \omega=\Delta \hat{\omega}$, and $N_{m}=N_{n}$, Eq. 21 becomes

$$
\tilde{x}(s)=\sum_{n=-N_{n} / 2}^{N_{n} / 2} \frac{A_{n} C_{n}}{\left(s-i \omega_{n}\right)^{2}}+\sum_{n=-N_{n} / 2}^{N_{n} / 2} \sum_{m=-N_{n} / 2, m \neq n}^{N_{n} / 2} \frac{A_{n} C_{m}}{\left(s-i \omega_{n}\right)\left(s-i \omega_{m}\right)}
$$

Thus, the pole-residue form of Eq. 26 is

$$
\tilde{x}(s)=\sum_{n=-N_{n} / 2}^{N_{n} / 2} \frac{A_{n} C_{n}}{\left(s-i \omega_{n}\right)^{2}}+\sum_{n=-N_{n} / 2}^{N_{n} / 2} \frac{P_{n}}{s-i \omega_{n}}
$$

where

$$
P_{n}=\sum_{m=-N_{n} / 2, m \neq n}^{N_{n} / 2} \frac{A_{n} C_{m}+A_{m} C_{n}}{i\left(\omega_{n}-\omega_{m}\right)}
$$

Taking the inverse Laplace transform of Eq. 27 gives

$$
x(t)=\sum_{n=-N_{n} / 2}^{N_{n} / 2} A_{n} C_{n} t \exp \left(i \omega_{n} t\right)+\sum_{n=-N_{n} / 2}^{N_{n} / 2} P_{n} \exp \left(i \omega_{n} t\right)
$$

Note that since the system and excitation share the same poles, one cannot separate the total response into the natural response and the forced response.

\section{RESPONSE CALCULATION IN THE LAPLACE DOMAIN}

As the pole-residue form of $\tilde{h}(s)$ in Eq. 17 has been based on designated pure imaginary poles, the system becomes a "periodic" oscillator with its IRF as a periodic function. This is certainly against the true physics, but the response calculation might be still valid for a period. To get a physically meaningful $\tilde{h}(s)$, one can search for the "true" poles $\mu_{n}$ of the system and the corresponding residues $\beta_{n}$. Consequently, the pole-residue form of $\tilde{h}(s)$ is expressed as

$$
\tilde{h}(s)=\sum_{n=1}^{N_{n}} \frac{\beta_{n}}{s-\mu_{n}}
$$

For extracting the true poles and residues of the system, a procedure shown schematically in Fig. 1 is proposed. First, taking the inverse Fourier transform of $\mathcal{H}(\omega)$ in Eq. 12 yields a real valued IRF, 
$h(t)$, for $t \geq 0$. While deriving $h(t)$ analytically is challenging, if not impossible, a straightforward way to compute a discrete IRF, $h\left(t_{k}\right)$, can be achieved by taking the inverse discrete Fourier transform (IDFT)—which can be efficiently carried out by using inverse fast Fourier transform (IFFT)—of a discretized FRF, $\mathcal{H}\left(\omega_{n}\right)$. After $h\left(t_{k}\right)$ has been computed, one applies the Prony-SS method (Hu et al. 2013) to approximate it as

$$
h(t) \approx \sum_{n=1}^{N_{n}} \beta_{n} \exp \left(\mu_{n} t\right)
$$

where $N_{n}$, the number of component, is usually a small integer, and $\beta_{n}$ and $\mu_{n}$ are constant coefficients. A summary of the Prony-SS method is given at Appendix I; its first step is to determine $N_{n}$. This is fulfilled by imposing a truncated singular value decomposition of a Hankel matrix built from the dealing signal $h\left(t_{k}\right)$, which has the similar concept to that of a principal component method. For a smooth signal, just a small number of $N_{n}$ is needed to achieve a good approximation. The method's second step is to compute $\mu_{n}$ obtained by the eigenvalue analysis of a realized matrix, and its last step is to compute $\beta_{n}$ based on solving a set of linear simultaneous equations. Because $h(t)$ is a real-valued function, $\mu_{n}$ must be either real numbers or in complex conjugate pairs. In turn, the coefficients $\beta_{n}$ must be also real or appear in complex conjugate pairs.

When $x(t)$ is computed based on Eq. 30, it is referred to as the Laplace domain approach in this paper, no matter how the input $f(t)$ has been decomposed. For the decomposition of $f(t)$, either a Prony series or a Fourier series can be used.

\section{Input decomposition: Prony series}

For an irregular excitation signal $f(t)$ of a finite duration of $T$, it can always be approximated by using the Prony-SS method (Hu et al. 2013):

$$
f(t)=\sum_{\ell=1}^{N_{\ell}} \alpha_{\ell} \exp \left(\lambda_{\ell} t\right) \quad 0 \leq t<T
$$

where $N_{\ell}$ is the number of components; $\alpha_{\ell}$ and $\lambda_{\ell}$ are constant coefficients. Taking the Laplace transform of Eq. (32) yields

$$
\tilde{f}(s)=\sum_{\ell=1}^{N_{\ell}} \frac{\alpha_{\ell}}{s-\lambda_{\ell}}
$$


From $\tilde{x}(s)=\tilde{h}(s) \tilde{f}(s)$, using Eqs. 30 and 33, one writes

$$
\tilde{x}(s)=\left(\sum_{n=1}^{N_{n}} \frac{\beta_{n}}{s-\mu_{n}}\right)\left(\sum_{\ell=1}^{N_{\ell}} \frac{\alpha_{\ell}}{s-\lambda_{\ell}}\right)
$$

Expressing Eq. 34 in a pole-residue form yields

$$
\tilde{x}(s)=\sum_{n=1}^{N_{n}} \frac{\gamma_{n}}{s-\mu_{n}}+\sum_{\ell=1}^{N_{\ell}} \frac{\kappa_{\ell}}{s-\lambda_{\ell}}
$$

where

$$
\gamma_{n}=\lim _{s \rightarrow \mu_{n}}\left(s-\mu_{n}\right) \tilde{x}(s)=\beta_{n} \tilde{f}\left(\mu_{n}\right)=\beta_{n}\left(\sum_{\ell=1}^{N_{\ell}} \frac{\alpha_{\ell}}{\mu_{n}-\lambda_{\ell}}\right)
$$

and

$$
\kappa_{\ell}=\lim _{s \rightarrow \lambda_{\ell}}\left(s-\lambda_{\ell}\right) \tilde{x}(s)=\alpha_{\ell} \tilde{h}\left(\lambda_{\ell}\right)=\frac{\alpha_{\ell}}{m \lambda_{\ell}^{2}+c\left(\lambda_{\ell}\right)^{q}+k}
$$

Performing the inverse Laplace transform to Eq. 35 then yields

$$
x(t)=\sum_{n=1}^{N_{n}} \gamma_{n} \exp \left(\mu_{n} t\right)+\sum_{\ell=1}^{N_{\ell}} \kappa_{\ell} \exp \left(\lambda_{\ell} t\right)
$$

In Eq. 38, the first sum term is the natural response governed by the system poles, $\mu_{n}$; and the second term is the forced response related to the excitation poles, $\lambda_{\ell}$.

\section{Input decomposition: Fourier series}

Similar to the formulation of Eq. 34, using Fourier series for $\tilde{f}(s)$, one writes

$$
\tilde{x}(s)=\left(\sum_{n=1}^{N_{n}} \frac{\beta_{n}}{s-\mu_{n}}\right)\left(\sum_{m=-N_{m} / 2}^{N_{m} / 2} \frac{C_{m}}{s-i \omega_{m}}\right)
$$

In its pole-residue form, one has:

$$
\tilde{x}(s)=\sum_{n=1}^{N_{n}} \frac{\delta_{n}}{s-\mu_{n}}+\sum_{m=-N_{m} / 2}^{N_{m} / 2} \frac{U_{m}}{s-i \omega_{m}}
$$

where

$$
\delta_{n}=\beta_{n} \tilde{f}\left(\mu_{n}\right)=\beta_{n}\left(\sum_{m=-N_{m} / 2}^{N_{m} / 2} \frac{C_{m}}{\mu_{n}-i \omega_{m}}\right)
$$


and

$$
U_{m}=C_{m} \tilde{h}\left(i \omega_{m}\right)=\frac{C_{m}}{-m \omega_{m}^{2}+c\left(i \omega_{m}\right)^{q}+k}
$$

So, taking the inverse Laplace transform of Eq. 40 gives

$$
x(t)=\sum_{n=1}^{N_{n}} \delta_{n} \exp \left(\mu_{n} t\right)+\sum_{m=-N_{m} / 2}^{N_{m} / 2} \mathcal{H}\left(\omega_{m}\right) C_{m} \exp \left(i \omega_{m} t\right)
$$

Note that although the natural responses of Eq. 38 and Eq. 43 share the same poles, they have different residues; consequently, Eqs. 38 and 43 have distinct natural responses. Clearly, the forced response in Eq. 43 is the steady-state response to the periodic loading. Theoretically, the total responses within $T$ for Eqs. 38 and 43 should be identical.

\section{NUMERICAL STUDIES}

A simple fractional oscillator with $q=1 / 2$ investigated in Ref. (Koh and Kelly 1990) is chosen in the numerical studies so that independent numerical verification for the computed response is possible; both simple sinusoidal and complicated earthquake loadings are considered. In addition, a fractional oscillator containing two irrational derivative terms excited by realistic earthquake motion will also be investigated to demonstrate a broader application of the proposed method.

Example 1: Fractional oscillator with $q=1 / 2$

The fractional oscillator considered first has $q=1 / 2$, with other values being mass $m=1$, stiffness $k=1$, and damping coefficient $c=0.1$, noting that throughout the numerical studies the unit system is the MKS (meter-kilogram-second) system. According to Eq. 12 and the chosen numerical quantities, the oscillator's FRF is:

$$
\mathcal{H}(\omega)=\frac{1}{-\omega^{2}+0.1 \times(i \omega)^{1 / 2}+1}
$$

\section{Sinusoidal loading}

Let the simple sinusoidal loading be $f(t)=\sin t$, which can be expressed as $f(t)=\alpha e^{\lambda t}+\bar{\alpha} e^{\bar{\lambda} t}$ where $\alpha=-0.5 i$ and $\lambda=i$. In other words, the chosen excitation has a pair of complex conjugate poles $\lambda, \bar{\lambda}= \pm i$ and the corresponding residues $\alpha, \bar{\alpha}=\mp 0.5 i$. 
Response calculation in the frequency domain While applying the proposed method in the frequency domain, the formulation of $\tilde{h}(s)$ is given in Eq. 17, whose poles are assigned at $i \hat{\omega}_{n}$ and residues $A_{n}=\mathcal{H}_{n} \Delta \hat{\omega} /(2 \pi)$ are computed directly from a discretized FRF (see Eq. 13). In the numerical implementation, Eq. 17 has been utilized with $\Delta \hat{\omega}=\pi / 256$ and $N_{n}=2048$. For the simple excitation $f(t)=\sin t$, while using Eq. 21, it has $N_{m}=2, \omega_{1}=1$, and $C_{1}=-0.5 i$. Plotted in Fig. 2 is the $x(t)$ computed based on Eq. 25; it agrees well with that obtained from the time domain method. As indicated in Eq. 25, the response $x(t)$ is composed of two terms, the natural response and the forced response. Fig. 3 plots these two terms separately. Note that the frequency domain method's natural response will also become periodic after $2 \pi / \Delta \hat{\omega}=512$.

Response calculation in the Laplace domain To apply the proposed method in the Laplace domain, one needs to follow the steps shown in Fig. 1 to obtain the pole-residue form of $\tilde{h}(s)$. To begin, a discretized FRF is generated from Eq. 44 with the frequency interval $\Delta \omega=\pi / 64$, number of frequency components $N_{n}=4096$, and cut-off frequency $\omega_{N}=64 \pi$. Then, performing the IFFT of this FRF to obtain a discrete IRF, $h\left(t_{k}\right)$, plotted in Fig. 4, which is in a good agreement with that reported in Ref. (Koh and Kelly 1990). Processing $h\left(t_{k}\right)$ by the Prony-SS method results in an approximated IRF, denoted $h_{a}(t)$, to be

$$
h_{a}(t)=\beta_{1} e^{\mu_{1} t}+\bar{\beta}_{1} e^{\bar{\mu}_{1} t}+\beta_{2} e^{\mu_{2} t}
$$

where $\mu_{1}=-0.0353-1.0353 i, \beta_{1}=-0.0055+0.4957 i, \mu_{2}=-0.2992$ and $\beta_{2}=0.0119$. Eq. 45 is plotted in Fig. 5, which indicates that the IRF is dominated by an oscillatory term (complex conjugate poles), together with a negligibly small non-oscillatory term (real pole). This feature concurs with the derivation in Refs. (Suarez and Shokooh 1995; Suarez and Shokooh 1997). In addition, the obtained $\mu_{1}$ value is nearly identical to that reported in Ref. (Suarez and Shokooh 1995). To evaluate the accuracy of Eq. 45 , the relative error $\epsilon_{h}\left(t_{k}\right)$ between $h\left(t_{k}\right)$ and $h_{a}\left(t_{k}\right)$, defined as

$$
\epsilon_{h}\left(t_{k}\right)=\frac{h_{a}\left(t_{k}\right)-h\left(t_{k}\right)}{\max \left[h\left(t_{k}\right)\right]}
$$


is computed and plotted in Fig. 6, which indicates the maximum of $\epsilon_{h}$ occurring at $t=0$ is only about $0.8 \%$. Furthermore, from Eq. 45 , the corresponding FRF is

$$
\mathcal{H}_{a}(\omega)=\frac{\beta_{1}}{i \omega-\mu_{1}}+\frac{\bar{\beta}_{1}}{i \omega-\bar{\mu}_{1}}+\frac{\beta_{2}}{i \omega-\mu_{2}}
$$

Shown in Fig. 7 is the comparison between Eqs. 44 and 47, including the magnitude in Fig. 7(a) and phase angle in Fig. 7(b). Indeed, they are in excellent agreement.

In this example, when the response functions $\tilde{x}(s)$ and $x(t)$ are expressed as Eq. 35 and Eq. 38 , respectively, there are 5 response poles, including two from the excitation poles and three system poles; the corresponding residues to the excitation and system poles are computed from Eqs. 36 and 37, respectively. The numerical values for all poles and residues are listed in Table 1. From them, the computed response $x(t)$ is shown in Fig. 8, which agrees well with the $x(t)$ that has been obtained by performing the numerical Duhamel integral based on the original $h(t)$. The natural response and forced response obtained from Eq. 38 are plotted in Fig. 9. As expected theoretically, they are completely different from those in Fig. 3. While the natural response diminishes with time due to the presence of the damping, the forced response remains sinusoidal with the frequency identical to the excitation frequency.

The proposed method is much more efficient in computational time than the Duhamel integral method, as shown in Fig. 10. In particular, the efficiency of the proposed method increases with the number of the time steps. As the system poles and residues are computed prior to the dynamic response calculation, the computational time for them are excluded in Fig. 10.

\section{Earthquake loading}

This example considers the oscillator characterized by Eq. 44 to earthquake-induced excitation $f(t)=-m \ddot{x}(t)$, where the chosen $\ddot{x}(t)$ is the measured E1 Centro earthquake acceleration signal in the EW direction, sampled with $\Delta t=0.02$ for $N_{s}=2048$ steps (see Fig. 11). In view of the complexity of this $f(t)$, the FFT algorithm is utilized to decompose it into a poleresidue form. The outcome of the FFT includes 1023 pairs of complex Fourier coefficients $C_{m}$ at $\omega_{m}=(2 \pi m) /\left(N_{s} \Delta t\right), m= \pm 1, \cdots, \pm 1023$, along with two real Fourier coefficients at zero and 
the Nyquist frequency, respectively. For the pole-residue form of $\tilde{f}(s), i \omega_{m}$ are the preselected excitation poles and $C_{m}$ are the corresponding residues.

Response calculation in the frequency domain While conducting the proposed method in the frequency domain, $\hat{\omega}_{n}$ in Eq. 17 , or $\Delta \hat{\omega}$, can be selected freely. Here, choosing $\Delta \hat{\omega}=\Delta \omega$ is for demonstrating the accuracy of the proposed method when it involves second-order poles. Using FFT automatically imposes the frequency resolution $\Delta \omega=2 \pi / T$ where $T$ is the signal duration. For increasing the frequency resolution, padding sufficient zeros at the end of the original signal has been a common technique. After padding zeros to triple the length of the excitation, the poles for both the excitation and system have been designated at $i \hat{\omega}_{n}=i(2 \pi n) /\left(3 N_{s} \Delta t\right)$, for $n=-3072, \cdots, 3071$. The required system residues $A_{n}=\mathcal{H}_{n} \Delta \hat{\omega} /(2 \pi)$ are calculated accordingly from the discretized FRF (see Eq. 13). From Eq. 29, the response $x(t)$ is computed, as shown in Fig. 12, which is in excellent agreement with that obtained by the Duhamel integral approach. For completeness, the two components of Eq. 29 are plotted in Fig. 13. Note that the computed $x(t)$ is only meaningful for $t<T=122.88$, which is 3 times the original earthquake signal length, due to the usages of "periodic" oscillator and loading. Actually, when $t$ becomes large, the computed $x(t)$ from Eq. 29 will diverge because the first term of Eq. 29 is linearly proportional to $t$.

Response calculation in the Laplace domain As the pole-residue forms of the excitation and system have been expressed as a Fourier series and Eq. 45, respectively, computing the response $x(t)$ in the Laplace domain is carried out by Eq. 43. Fig. 14 shows the obtained $x(t)$, which is in excellent agreement with that computed by the Duhamel integral in the time domain. For completeness, the natural response and the steady-state response are also plotted in Fig. 15.

Example 2: Fractional oscillator with two irrational fractional derivative terms: $q_{1}=\pi / 30$ and $q_{2}=2 \pi / 7$

Unlike most traditional methods, the proposed frequency/Laplace methods is not limited to oscillators with rational fractional orders. To demonstrate its broader application, the oscillator considered in this example contains two fractional derivative terms: $q_{1}=\pi / 30$ and $q_{2}=2 \pi / 7$. 
Let other parameters be $m=5 / 2, k=8 / 5, c_{1}=1 / 2$, and $c_{2}=6 / 5$; as a result, the oscillator's FRF is:

$$
\mathcal{H}(\omega)=\frac{1}{-5 / 2 \times \omega^{2}+1 / 2 \times(i \omega)^{\pi / 30}+6 / 5 \times(i \omega)^{2 \pi / 7}+8 / 5}
$$

In the frequency domain approach, the poles assigned at $i \hat{\omega}_{n}$ and residues $A_{n}=\mathcal{H}_{n} \Delta \hat{\omega} /(2 \pi)$ are computed based on $\Delta \hat{\omega}=\pi / 256$ and $N_{n}=2048$. The same numerical verifications conducted in previous examples are repeated here. Fig. 16, which plots the response caused by the same earthquake loading utilized in the previous example, shows a good agreement between the proposed method and the Duhamel method. For completeness, the two components of Eq. 29 are also plotted in Fig. 17.

To apply the Laplace domain approach, the pole-residue form of $\tilde{h}(s)$ with true poles must be determined first. As this method approximates a pole-residue form for $h_{a}(t)$ by the data-driven Prony-SS method, its implementation will not be affected by whether the fractional derivatives are rational or irrational. Conducting the same procedure yields the approximated IRF with $\mu_{1}=$ $-0.2899 \mp 1.0081 i, \beta_{1}=-0.0188 \pm 0.2230 i, \mu_{2}=-0.1352$ and $\beta_{2}=0.0246$; the two components of $h_{a}(t)$ are plotted in Fig. 18. Furthermore, Fig. 19 shows a good agreement between the original IRF and the reconstructed $h_{a}(t)$. Lastly, the response due to the earthquake loading computed by the Laplace domain approach is shown in Fig. 20, which has been verified by the Duhamel integral approach.

\section{CONCLUDING REMARKS}

A pole-residue method, operated in either the frequency or the Laplace domain, to efficiently compute the response of a fractional oscillator (FO) to complicated irregular loadings was developed and tested. Unlike many existing approaches that had strict limitations on the fractional order $q$, the proposed method was applicable to any $q$ between 0 and 1 . Despite the fact that the exact transfer function (TF) and frequency response function (FRF) of an FO had been derived analytically, using them directly to compute the FO's transient response was not possible. This paper developed two distinct approaches to convert the exact TF into its pole-residue form. 
The first approach was a frequency domain method that designated "artificial" poles along the imaginary axis. The second approach was a Laplace domain method that extracted the "ture" poles through two steps: (1) a discrete IRF was produced by taking the inverse Fourier transform of the corresponding FRF that was readily obtained from the exact TF; (2) a complex exponential signal decomposition method, i.e., the Prony-SS method, was invoked to extract the poles and residues. Through two numerical examples with oscillators involving rational and irrational derivatives, respectively, the correctness of the proposed method operated in both the frequency and Laplace domains was verified by a time-consuming numerical time domain method.

\section{ACKNOWLEDGEMENT}

The research was financially supported by the Postdoctoral Research Foundation of China (Grant No.2021M690521) and the National Natural Science Foundation of China (Grant No.51879250).

\section{APPENDIX I: SUMMARY OF THE PRONY-SS METHOD}

Given the IRF signal $h(t)$ that are sampled with interval $\Delta t, h\left(t_{k}\right)=h(k \Delta t), k=$ $0,1, \cdots, N-1$, using the Prony-SS method is to approximate $h(t)$ as $\sum_{n=1}^{N_{n}} \beta_{n} \exp \left(\mu_{n} t\right)$. Summarized here is the three sequential steps of the Prony-SS method to determine $N_{n}, \mu_{n}$ and $\beta_{n}$, respectively.

Step 1 for determining $N_{n}$ : Construct the Hankel matrix $\mathbf{H}(0) \in \mathbb{R}^{\xi \times \eta}$ from the sampled signal $h\left(t_{k}\right)$ :

$$
\mathbf{H}(0)=\left[\begin{array}{cccc}
h\left(t_{0}\right) & h\left(t_{1}\right) & \cdots & h\left(t_{\eta-1}\right) \\
h\left(t_{1}\right) & h\left(t_{2}\right) & \cdots & h\left(t_{\eta}\right) \\
\vdots & \vdots & \ddots & \vdots \\
h\left(t_{\xi-1}\right) & h\left(t_{\xi}\right) & \cdots & h\left(t_{\xi+\eta-2}\right)
\end{array}\right]
$$

where $\xi+\eta=N$, and a better choice is both $\xi$ and $\eta$ are close to $N / 2$. Applying the singular value decomposition of $\mathbf{H}(0)$ gives

$$
\mathbf{H}(0)=\left[\begin{array}{ll}
\mathbf{U}_{1} & \mathbf{U}_{2}
\end{array}\right]\left[\begin{array}{cc}
\mathbf{S}_{1} & \mathbf{0} \\
\mathbf{0} & \mathbf{0}
\end{array}\right]\left[\begin{array}{c}
\mathbf{V}_{1}^{T} \\
\mathbf{V}_{2}^{T}
\end{array}\right]=\mathbf{U}_{1} \mathbf{S}_{1} \mathbf{V}_{1}^{T}
$$


Where $\mathbf{U}_{1} \in \mathbb{R}^{\xi \times N_{n}}, \mathbf{S}_{1} \in \mathbb{R}^{N_{n} \times N_{n}}$ and $\mathbf{V}_{1} \in \mathbb{R}^{\eta \times N_{n}}$; theoretically, $N_{n}$ is the rank of $\mathbf{H}(0)$, i.e., the number of non-zero singular values in Eq. 50. Mathematically, the singular values go to zero when the rank of the matrix is exceeded; however, for data involving random errors or inconsistencies, some singular values will be very small, but not exactly zero. In this situation, $N_{n}$ could be determined based on the magnitudes of the singular values having been ordered sequentially from the largest to the smallest; a conventional way to determine $N_{n}$ is based on a significant drop of the normalized singular values.

Step 2 for determining $\mu_{n}$ : Construct the Hankel matrix $\mathbf{H}(1) \in \mathbb{R}^{\xi \times \eta}$ from the sampled signal $h\left(t_{k}\right)$ :

$$
\mathbf{H}(1)=\left[\begin{array}{cccc}
h\left(t_{1}\right) & h\left(t_{2}\right) & \cdots & h\left(t_{\eta}\right) \\
h\left(t_{2}\right) & h\left(t_{3}\right) & \cdots & h\left(t_{1+\eta}\right) \\
\vdots & \vdots & \ddots & \vdots \\
h\left(t_{\xi}\right) & h\left(t_{1+\xi}\right) & \cdots & h\left(t_{\xi+\eta-1}\right)
\end{array}\right]
$$

Using $\mathbf{U}_{1}, \mathbf{S}_{1}, \mathbf{V}_{1}$ and $\mathbf{H}(1)$, one computes

$$
\mathbf{A}=\mathbf{S}_{1}^{-1 / 2} \mathbf{U}_{1}^{T} \mathbf{H}(1) \mathbf{V}_{1} \mathbf{S}_{1}^{-1 / 2}
$$

After computing the eigenvalues $z_{n}, n=1, \cdots, N_{n}$, of $\mathbf{A}$, one can get $\mu_{n}=\ln \left(z_{n}\right) / \Delta t$.

Step 3 for determining $\beta_{n}$ : Compute the complex coefficients $\beta_{n}$ by solving a set of linear equations using a least-square procedure, based on the obtained $z_{n}$ and the sampled $h\left(t_{k}\right)$ :

$$
\left[\begin{array}{cccc}
z_{1}^{0} & z_{2}^{0} & \cdots & z_{N_{n}}^{0} \\
z_{1}^{1} & z_{2}^{1} & \cdots & z_{N_{n}}^{1} \\
\vdots & \vdots & \ddots & \vdots \\
z_{1}^{N-1} & z_{2}^{N-1} & \cdots & z_{N_{n}}^{N-1}
\end{array}\right]\left\{\begin{array}{c}
\beta_{1} \\
\beta_{2} \\
\vdots \\
\beta_{N_{n}}
\end{array}\right\}=\left\{\begin{array}{c}
h\left(t_{0}\right) \\
h\left(t_{1}\right) \\
\vdots \\
h\left(t_{N-1}\right)
\end{array}\right\}
$$

\section{DECLARATIONS}

\section{Funding}

- Postdoctoral Research Foundation of China, Grant No.2021M690521, received by Qianying $\mathrm{Cao}$ 
- National Natural Science Foundation of China, Grant No.51879250, received by Huajun $\mathrm{Li}$

\section{Conflicts of interest}

The authors declare that they have no known competing financial interests or personal relationships that could have appeared to influence the work reported in this paper.

\section{Availability of data and material}

All data and material that support the findings of this study are available from the corresponding author by email (jameshu@uri.edu).

\section{Code availability}

All code that support the findings of this study are available from the corresponding author by email (jameshu@uri.edu).

\section{Authors' contributions}

- Qianying Cao: Conceptualization, Methodology, Formal analysis, Software, Validation, Writing-Original draft, Funding acquisition

- Sau-Lon James Hu: Conceptualization, Methodology, Supervision, Writing-Review \& Editing, Project administration

- Huajun Li: Resources, Review \& editing, Funding acquisition

\section{Ethics approval}

Not applicable.

\section{Consent to participate}

Not applicable.

\section{Consent for publication}

Not applicable.

\section{REFERENCES}


Achar, B., Hanneken, J., and Clarke, T. (2002). "Response characteristics of a fractional oscillator." Physica A: Statistical Mechanics and its Applications, 309(3-4), 275-288.

Achar, B., Hanneken, J., and Clarke, T. (2004). "Damping characteristics of a fractional oscillator." Physica A: Statistical Mechanics and its Applications, 339(3-4), 311-319.

Agrawal, O. (1999). "An analytical scheme for stochastic dynamic systems containing fractional derivatives.” Proceedings of the 1999 ASME Design Engineering Technical Conferences.

Agrawal, O. (2001). "Stochastic analysis of dynamic systems containing fractional derivatives." Journal of Sound and Vibration, 5(247), 927-938.

Bagley, R. and Calico, R. (1985). "The fractional order state equations for the control of viscoelastically damped structures.” AIAA journal, 23(6), 918-925.

Bagley, R. and Torvik, J. (1983). "Fractional calculus-a different approach to the analysis of viscoelastically damped structures.” AIAA Journal, 21(5), 741-748.

Caputo, M. (1967). "Linear models of dissipation whose q is almost frequency independenttii." Geophysical Journal International, 13(5), 529-539.

Chang, T. and Singh, M. (2002). "Seismic analysis of structures with a fractional derivative model of viscoelastic dampers." Earthquake Engineering and Engineering Vibration, 1(2), 251-260.

Cortés, F. and Elejabarrieta, M. (2007). "Finite element formulations for transient dynamic analysis in structural systems with viscoelastic treatments containing fractional derivative models." International Journal for Numerical Methods in Engineering, 69(10), 2173-2195.

Craig, R. and Kurdila, A. (2006). Fundamentals of structural dynamics. John Wiley \& Sons.

Di Paola, M., Failla, G., and Pirrotta, A. (2012). "Stationary and non-stationary stochastic response of linear fractional viscoelastic systems." Probabilistic Engineering Mechanics, 28, 85-90.

Gaul, L., Klein, P., and Kempfle, S. (1989). "Impulse response function of an oscillator with fractional derivative in damping description.” Mechanics Research Communications, 16(5), 297-305.

$\mathrm{Hu}, \mathrm{S}$. and Gao, B. (2018). "Computing transient response of dynamic systems in the frequency domain.” Journal of Engineering Mechanics, 144(2), 04017167. 
Hu, S., Liu, F., Gao, B., and Li, H. (2016). "Pole-residue method for numerical dynamic analysis." Journal of Engineering Mechanics, 142(8), 04016045.

Hu, S., Yang, W., and Li, H. (2013). "Signal decomposition and reconstruction using complex exponential models.” Mechanical Systems and Signal Processing, 40(2), 421-438.

Huang, Z., Jin, X., Lim, C. W., and Wang, Y. (2010). "Statistical analysis for stochastic systems including fractional derivatives.” Nonlinear Dynamics, 59(1-2), 339-349.

Koh, C. and Kelly, J. (1990). "Application of fractional derivatives to seismic analysis of baseisolated models.” Earthquake Engineering \& Structural Dynamics, 19(2), 229-241.

Kreyszig, E. (2010). Advanced engineering mathematics. John Wiley \& Sons.

Matlob, M. and Jamali, Y. (2019). "The concepts and applications of fractional order differential calculus in modeling of viscoelastic systems: A primer." Critical Reviews in Biomedical Engineering, 47(4).

Mendiguren, J., Cortés, F., and Galdos, L. (2012). "A generalised fractional derivative model to represent elastoplastic behaviour of metals." International Journal of Mechanical Sciences, 65(1), 12-17.

Naber, M. (2010). "Linear fractionally damped oscillator.” International Journal of Differential Equations.

Pinnola, F. (2016). "Statistical correlation of fractional oscillator response by complex spectral moments and state variable expansion." Communications in Nonlinear Science and Numerical Simulation, 39, 343-359.

Sheng, H., Li, Y., and Chen, Y. (2011). "Application of numerical inverse laplace transform algorithms in fractional calculus." Journal of the Franklin Institute, 348(2), 315-330.

Shokooh, A. and Suárez, L. (1999). "A comparison of numerical methods applied to a fractional model of damping materials.” Journal of Vibration and Control, 5(3), 331-354.

Suarez, L. and Shokooh, A. (1995). "Response of systems with damping materials modeled using fractional calculus.” Applied Mechanics Review, 48(11).

Suarez, L. and Shokooh, A. (1997). "An eigenvector expansion method for the solution of motion 
containing fractional derivatives.” Journal of Applied Mechanics, 64(3), 629-635.

Ye, K., Li, L., and Tang, J. (2003). "Stochastic seismic response of structures with added viscoelastic dampers modeled by fractional derivative." Earthquake Engineering and Engineering Vibration, 2(1), 133-139.

Yuan, L. and Agrawal, O. (2002). "A numerical scheme for dynamic systems containing fractional derivatives.” Journal of Vibration and Acoustics, 124(2), 321-324.

Zarraga, O., Sarría, I., García-Barruetabeña, J., and Cortés, F. (2019). "An analysis of the dynamical behaviour of systems with fractional damping for mechanical engineering applications.” Symmetry, 11(12), 1499. 
List of Tables

1 Computed poles and residues of the loading, transfer function and displace- 
Table 1. Computed poles and residues of the loading, transfer function and displacement response

\begin{tabular}{|l|l|l|}
\hline & Poles & Residues \\
\hline Loading & $\lambda, \bar{\lambda}= \pm i$ & $\alpha, \bar{\alpha}=\mp 0.5 i$ \\
\hline \multirow{2}{*}{ Transfer function } & $\mu_{1}, \bar{\mu}_{1}=-0.0353 \mp 1.0353 i$ & $\beta_{1}, \bar{\beta}_{1}=-0.0055 \pm 0.4957 i$ \\
& $\mu_{2}=-0.2992$ & $\beta_{2}=0.0119$ \\
\hline \multirow{2}{*}{ Response } & $\lambda, \bar{\lambda}= \pm i$ & $\kappa, \bar{\kappa}=-3.5355 \mp 3.5355 i$ \\
& $\mu_{1}, \bar{\mu}_{1}=-0.0353 \mp 1.0353 i$ & $\gamma_{1}, \bar{\gamma}_{1}=3.5423 \mp 3.3457 i$ \\
& $\mu_{2}=-0.2992$ & $\gamma_{2}=0.0110$ \\
\hline
\end{tabular}




\section{List of Figures}

1 Computing the corresponding pole-residue form for $\tilde{h}(s)=1 /\left(m s^{2}+c s^{q}+k\right) 26$

2 Response of the SDOF fractional oscillator with $q=1 / 2$ to sinusoidal loading based on the frequency domain and the Duhamel integral methods . 27

3 Natural and forced responses of the SDOF fractional oscillator with $q=$ $1 / 2$ to sinusoidal loading by the frequency domain method . . . . . . . . 28

4 Response of the SDOF fractional oscillator with $q=1 / 2$ due to the unit impulsive excitation . . . . . . . . . . . . . . . . . . . 29

5 Two components of the IRF for oscillator with $q=1 / 2$ : (a) dominated oscillatory term; (b) minor non-oscillatory term . . . . . . . . . . . . 30

6 The SDOF fractional oscillator with $q=1 / 2$ : (a) Comparison between $h(t)$ and $h_{a}(t) ;(\mathrm{b})$ Relative error between $h(t)$ and $h_{a}(t) \ldots 31$

7 Comparison of $\mathcal{H}(\omega)$ and $\mathcal{H}_{a}(\omega)$ for oscillator with $q=1 / 2$ : (a) the magnitude $\left|\mathcal{H}_{a}(\omega)\right| ;$ (b) the phase $\theta \ldots \ldots . \ldots . \ldots 32$

8 Response of the SDOF fractional oscillator with $q=1 / 2$ to sinusoidal loading based on the Laplace domain and the Duhamel integral methods . . 33

9 Natural and forced responses of the SDOF fractional oscillator with $q=$ $1 / 2$ to sinusoidal loading by the Laplace domain method . . . . . . . . . . 34

10 Comparison of computation efficiency of the Laplace method and the Duhamel integral method . . . . . . . . . . . . . . . 35

11 The measured E1 Centro earthquake acceleration signal . . . . . . . . . . 36

12 Response of the SDOF fractional oscillator with $q=1 / 2$ to the earthquake loading based on the frequency domain and Duhamel integral methods . . . 37

13 Two response components of the SDOF fractional oscillator with $q=1 / 2$ to the earthquake loading by the frequency domain method . . . . . . . . 38

14 Response of the SDOF fractional oscillator with $q=1 / 2$ to the earthquake loading based on the Laplace domain and the Duhamel integral methods . . 39 
15 Natural and forced responses of the SDOF fractional oscillator with $q=$ $1 / 2$ to the earthquake loading by the Laplace domain method . . . . . . . . 40

16 Response of the SDOF fractional oscillator with $q_{1}=\pi / 30$ and $q_{2}=2 \pi / 7$ to the earthquake loading based on the frequency domain and Duhamel integral methods . . . . . . . . . . . . . . . . . . . . 41

17 Two response components of the SDOF fractional oscillator with $q_{1}=$ $\pi / 30$ and $q_{2}=2 \pi / 7$ to the earthquake loading by the frequency domain

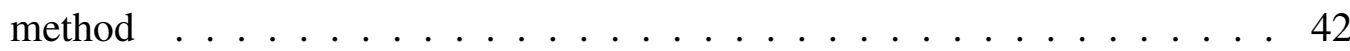

18 Two components of the IRF for oscillator with $q_{1}=\pi / 30$ and $q_{2}=2 \pi / 7$ :

(a) dominated oscillatory term; (b) minor non-oscillatory term . . . . . . . 43

19 The SDOF fractional oscillator with $q_{1}=\pi / 30$ and $q_{2}=2 \pi / 7$ : (a) Comparison between $h(t)$ and $h_{a}(t)$; (b) Relative error between $h(t)$ and

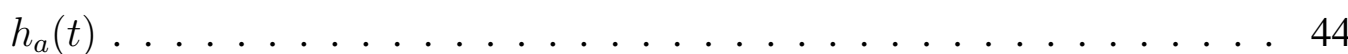

20 Response of the SDOF fractional oscillator with $q_{1}=\pi / 30$ and $q_{2}=2 \pi / 7$ to the earthquake loading based on the Laplace domain and Duhamel integral methods . . . . . . . . . . . . . . . . . . . 4 


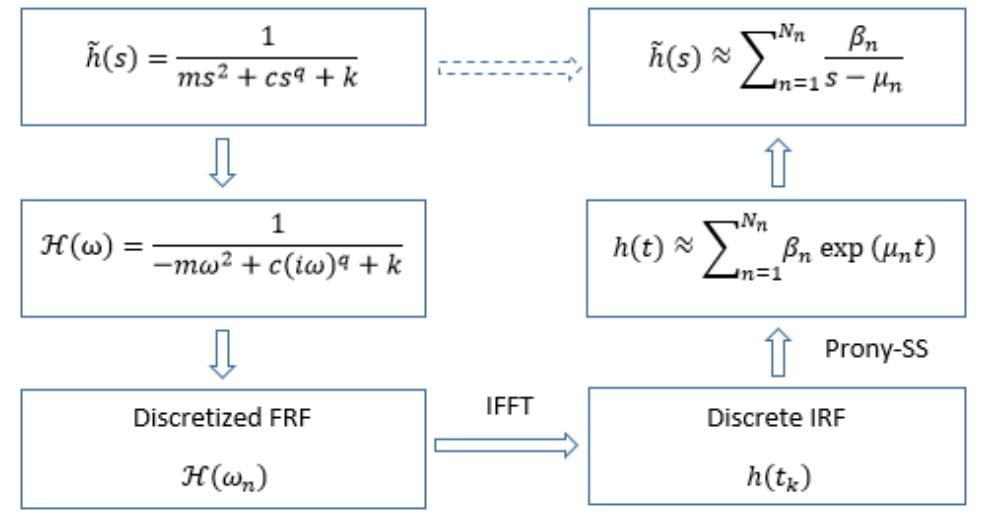

Fig. 1. Computing the corresponding pole-residue form for $\tilde{h}(s)=1 /\left(m s^{2}+\right.$ $\left.c s^{q}+k\right)$ 


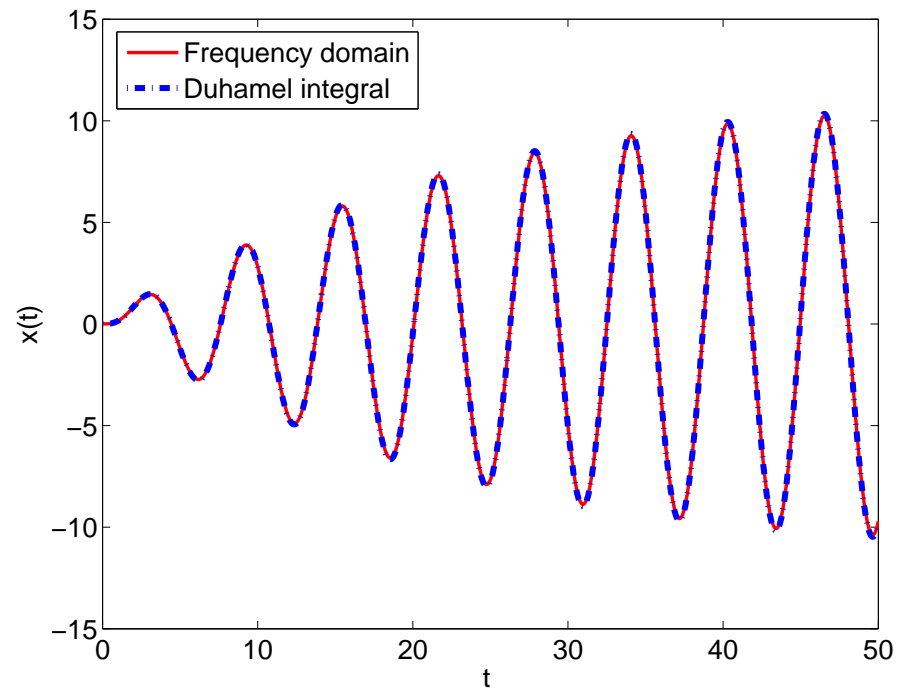

Fig. 2. Response of the SDOF fractional oscillator with $q=1 / 2$ to sinusoidal loading based on the frequency domain and the Duhamel integral methods 


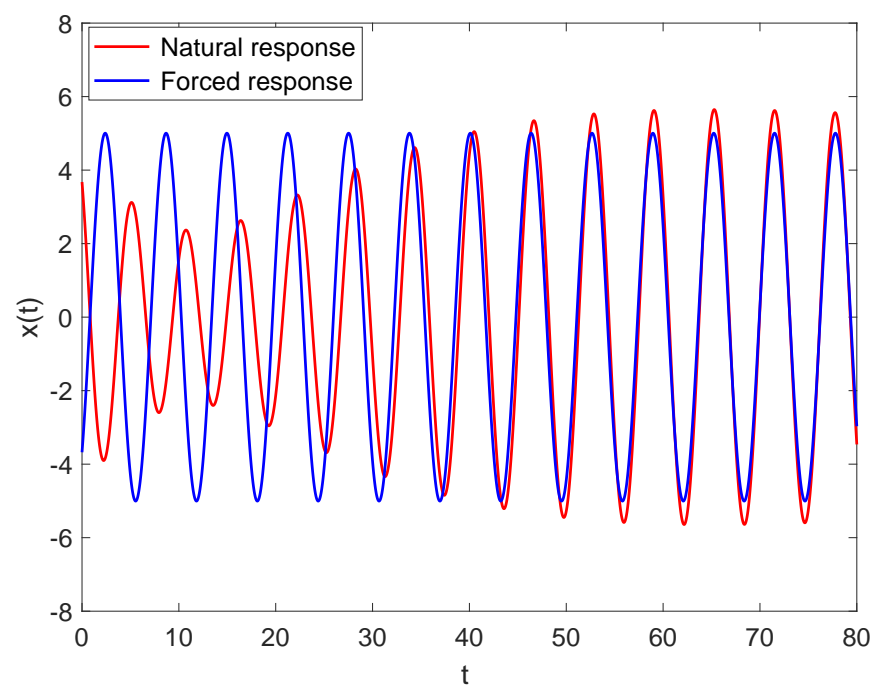

Fig. 3. Natural and forced responses of the SDOF fractional oscillator with $q=1 / 2$ to sinusoidal loading by the frequency domain method 


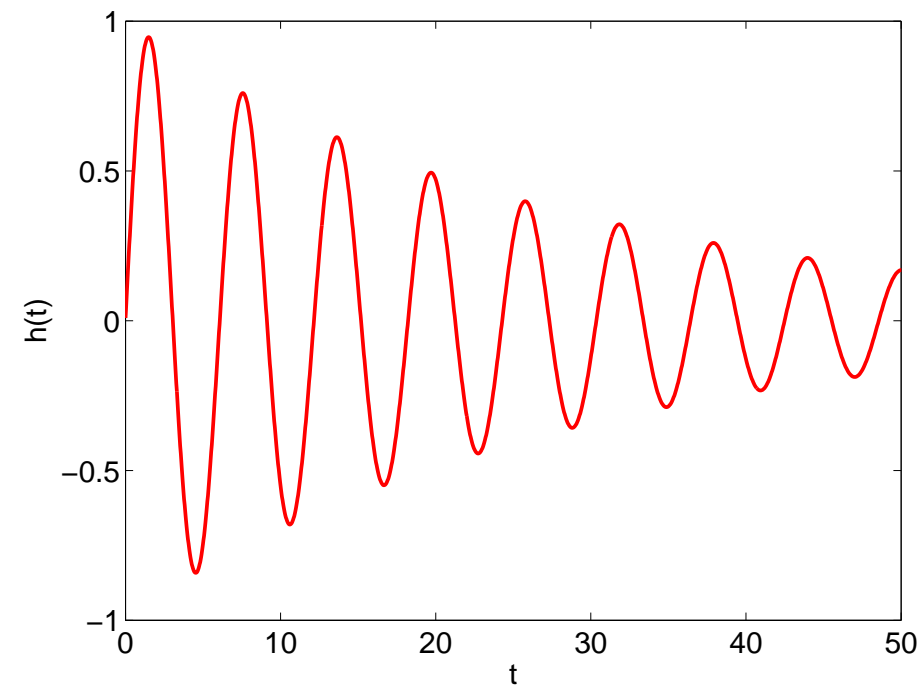

Fig. 4. Response of the SDOF fractional oscillator with $q=1 / 2$ due to the unit impulsive excitation 

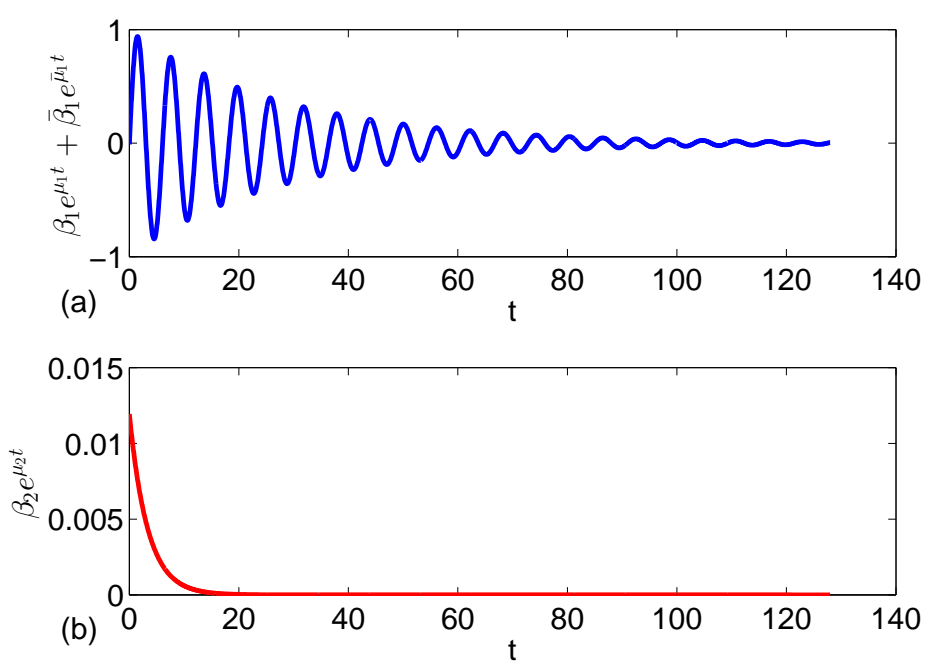

Fig. 5. Two components of the IRF for oscillator with $q=1 / 2$ : (a) dominated oscillatory term; (b) minor non-oscillatory term 

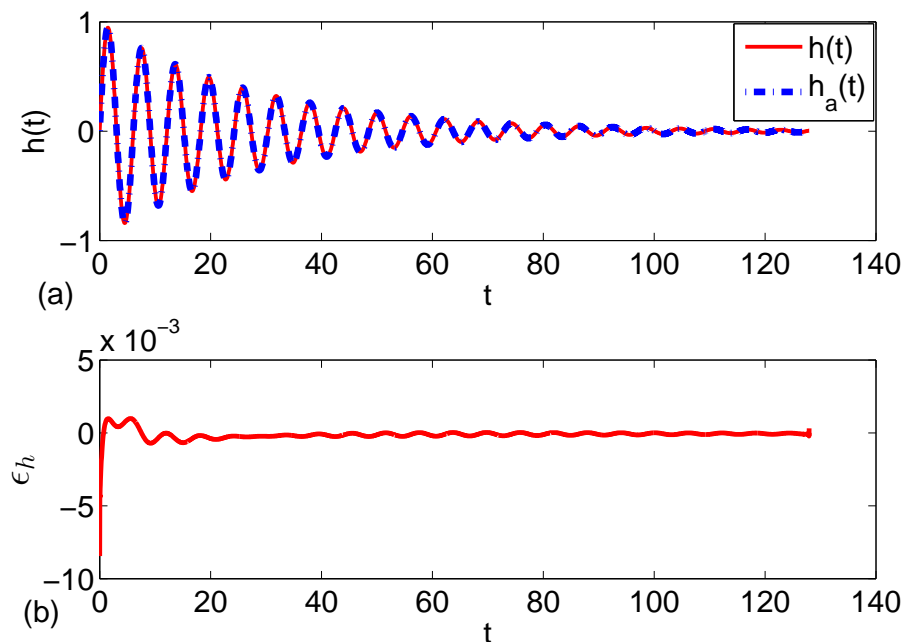

Fig. 6. The SDOF fractional oscillator with $q=1 / 2$ : (a) Comparison between $h(t)$ and $h_{a}(t)$; (b) Relative error between $h(t)$ and $h_{a}(t)$ 

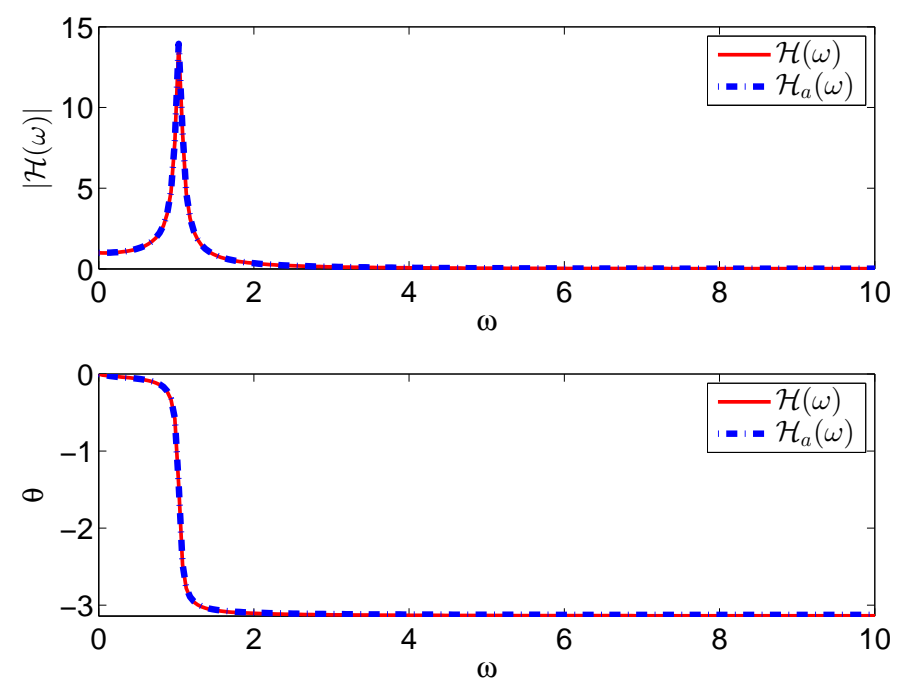

Fig. 7. Comparison of $\mathcal{H}(\omega)$ and $\mathcal{H}_{a}(\omega)$ for oscillator with $q=1 / 2$ : (a) the magnitude $\left|\mathcal{H}_{a}(\omega)\right|$; (b) the phase $\theta$ 


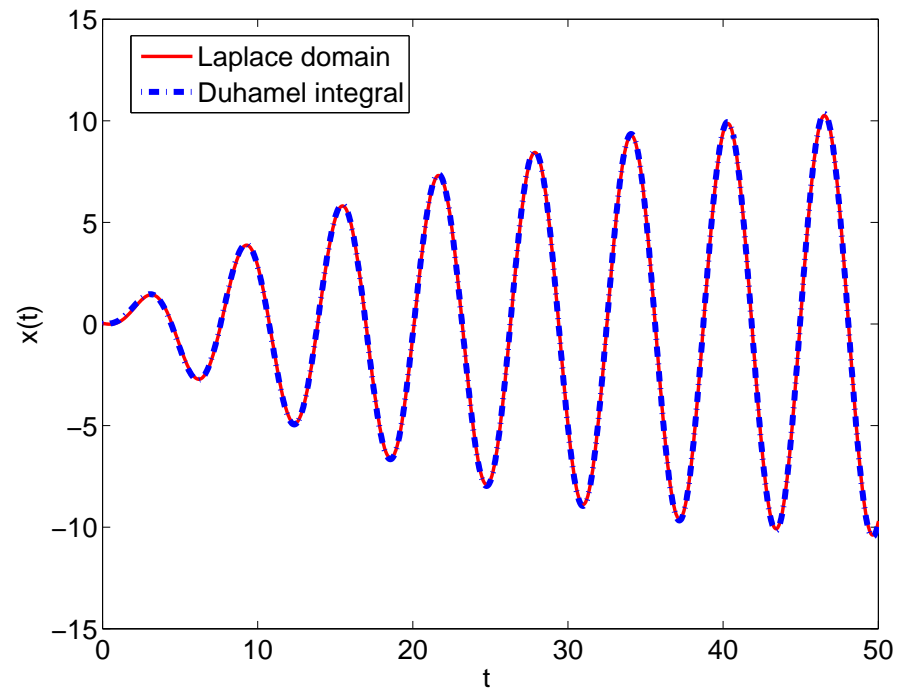

Fig. 8. Response of the SDOF fractional oscillator with $q=1 / 2$ to sinusoidal loading based on the Laplace domain and the Duhamel integral methods 


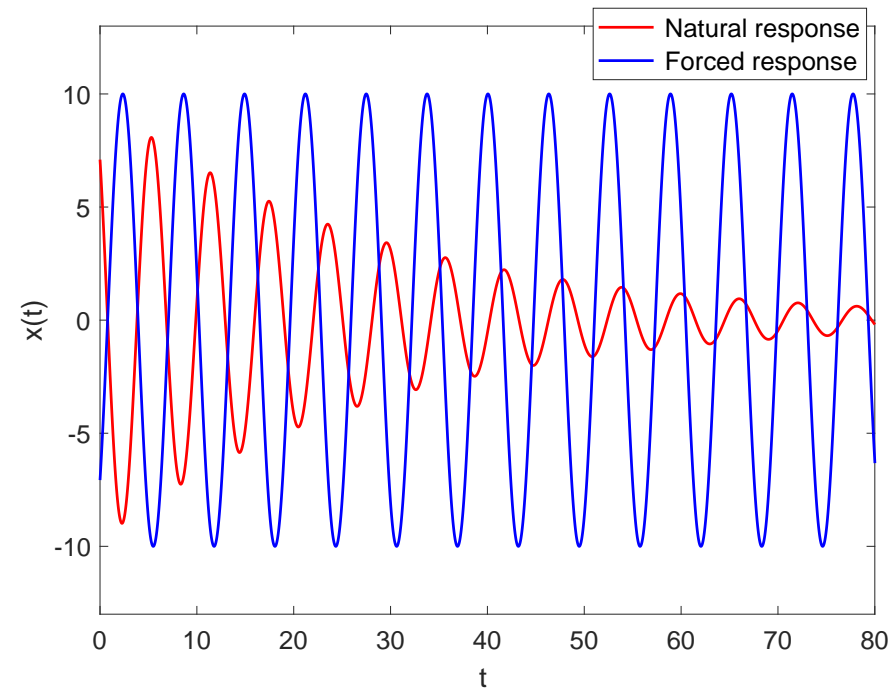

Fig. 9. Natural and forced responses of the SDOF fractional oscillator with $q=1 / 2$ to sinusoidal loading by the Laplace domain method 


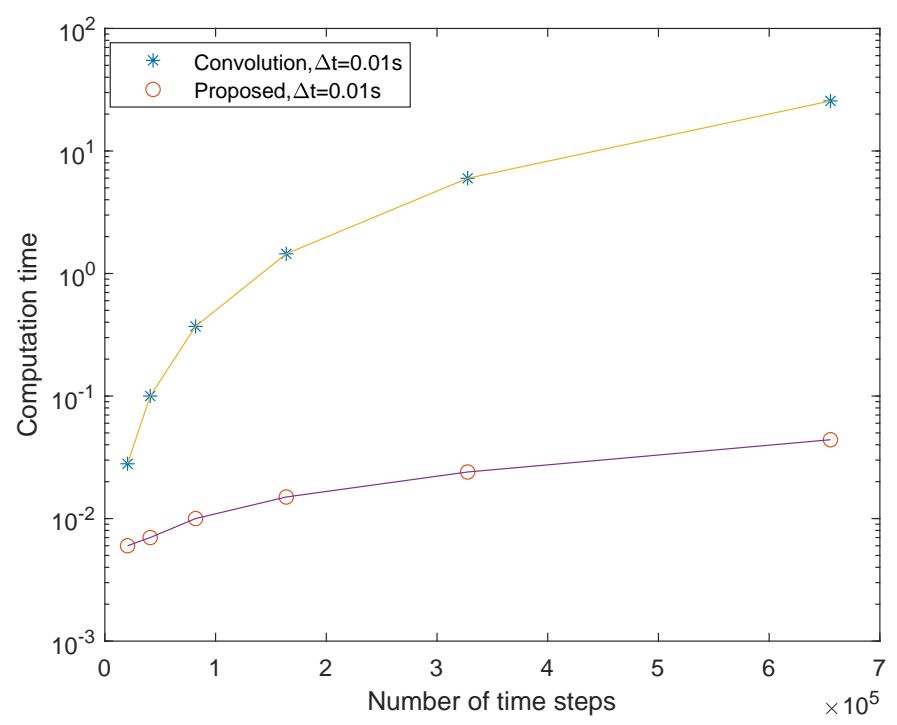

Fig. 10. Comparison of computation efficiency of the Laplace method and the Duhamel integral method 


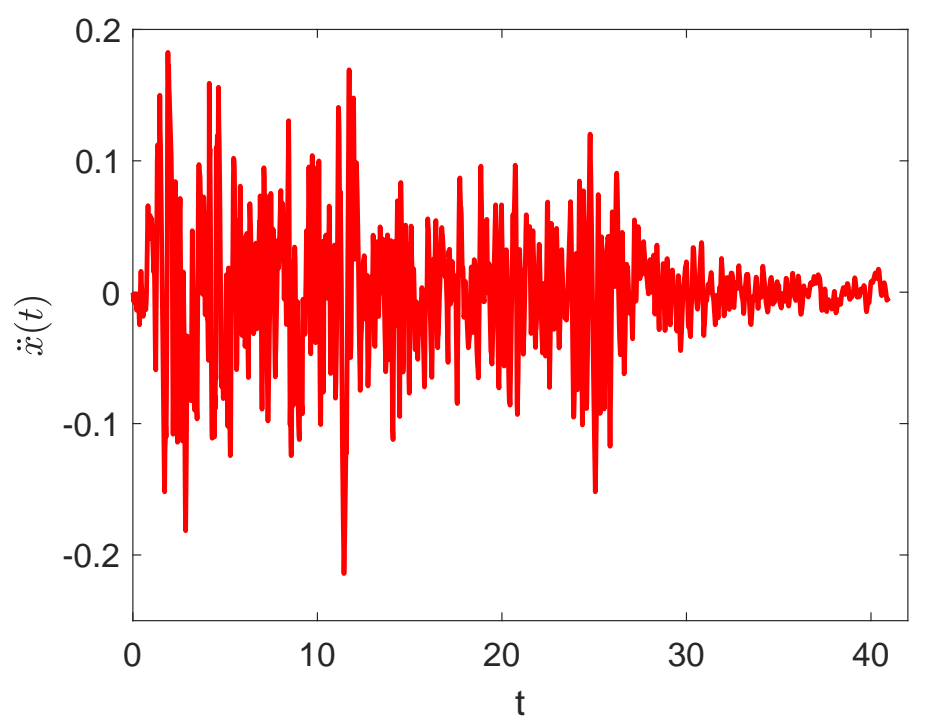

Fig. 11. The measured E1 Centro earthquake acceleration signal 


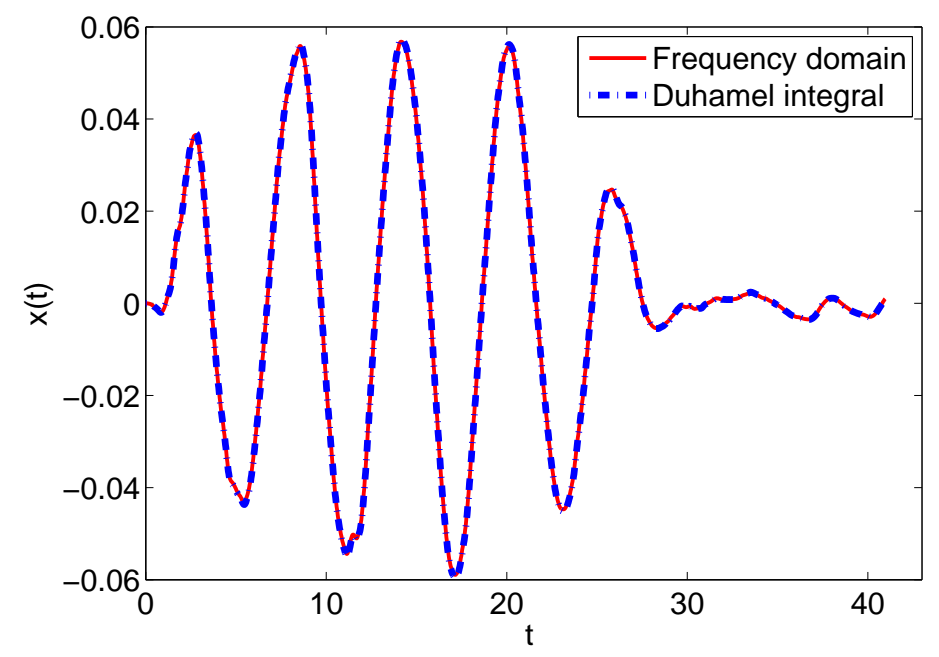

Fig. 12. Response of the SDOF fractional oscillator with $q=1 / 2$ to the earthquake loading based on the frequency domain and Duhamel integral methods 


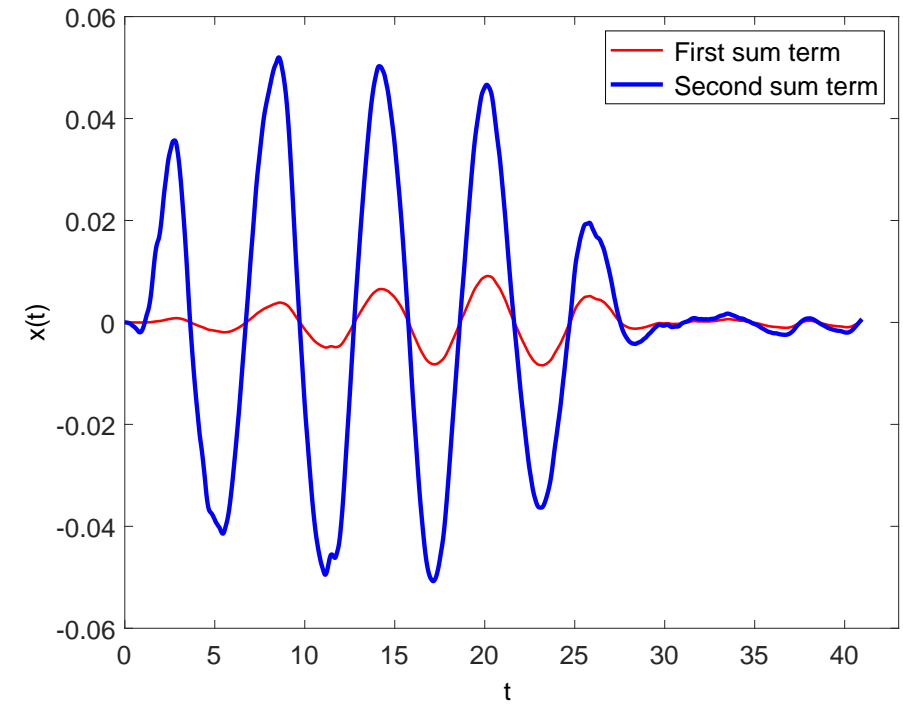

Fig. 13. Two response components of the SDOF fractional oscillator with $q=1 / 2$ to the earthquake loading by the frequency domain method 


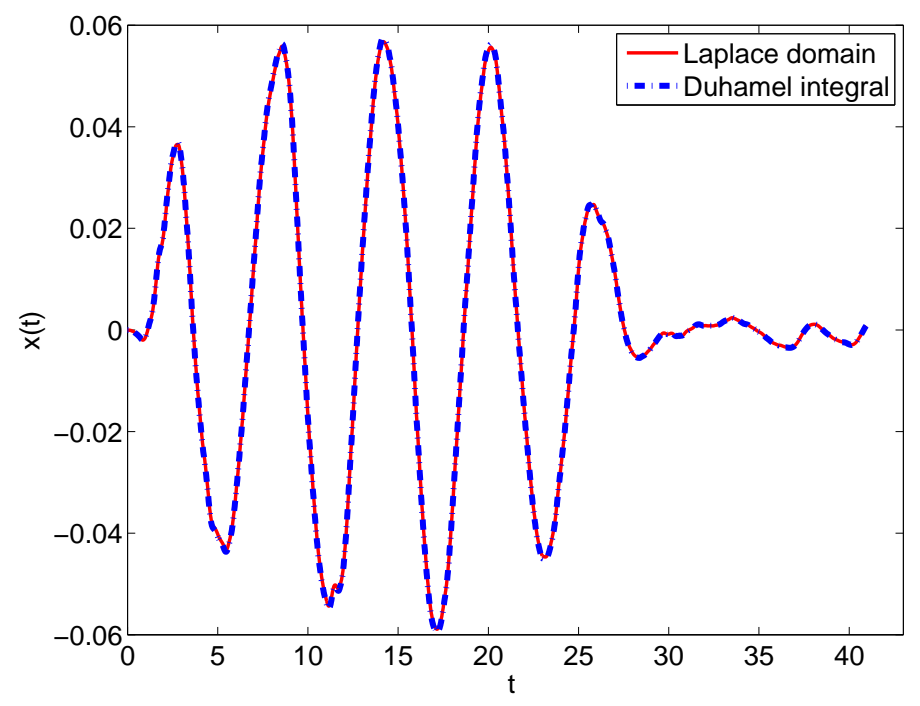

Fig. 14. Response of the SDOF fractional oscillator with $q=1 / 2$ to the earthquake loading based on the Laplace domain and the Duhamel integral methods 


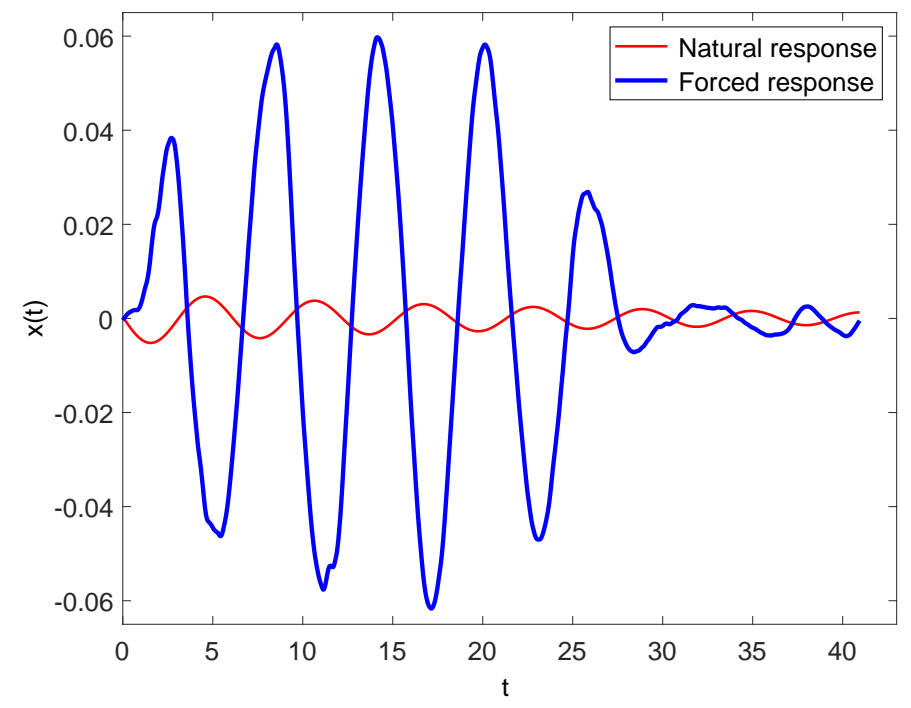

Fig. 15. Natural and forced responses of the SDOF fractional oscillator with $q=1 / 2$ to the earthquake loading by the Laplace domain method 


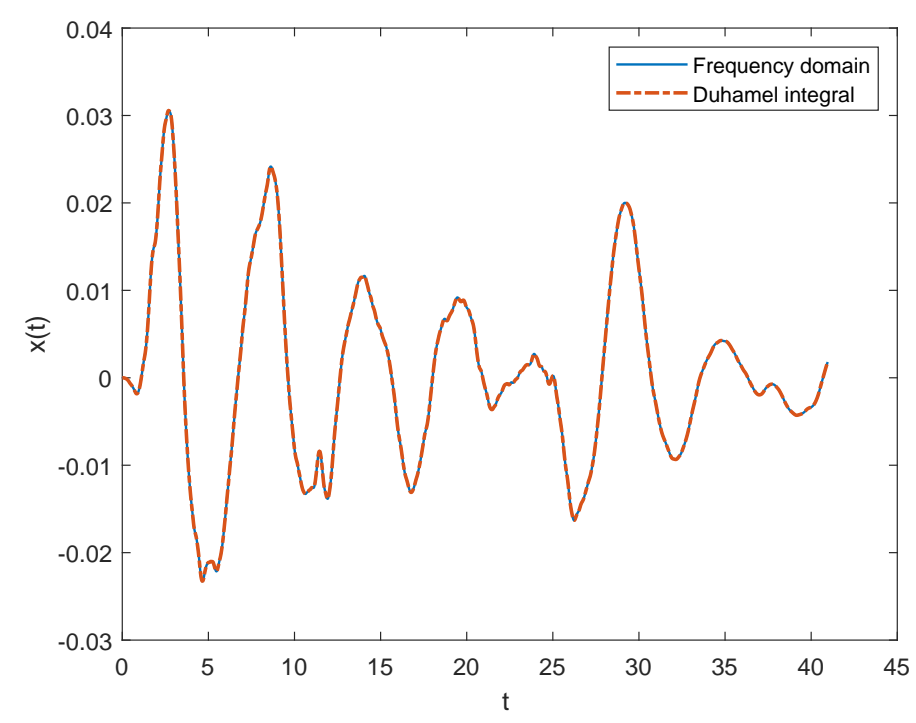

Fig. 16. Response of the SDOF fractional oscillator with $q_{1}=\pi / 30$ and $q_{2}=$ $2 \pi / 7$ to the earthquake loading based on the frequency domain and Duhamel integral methods 


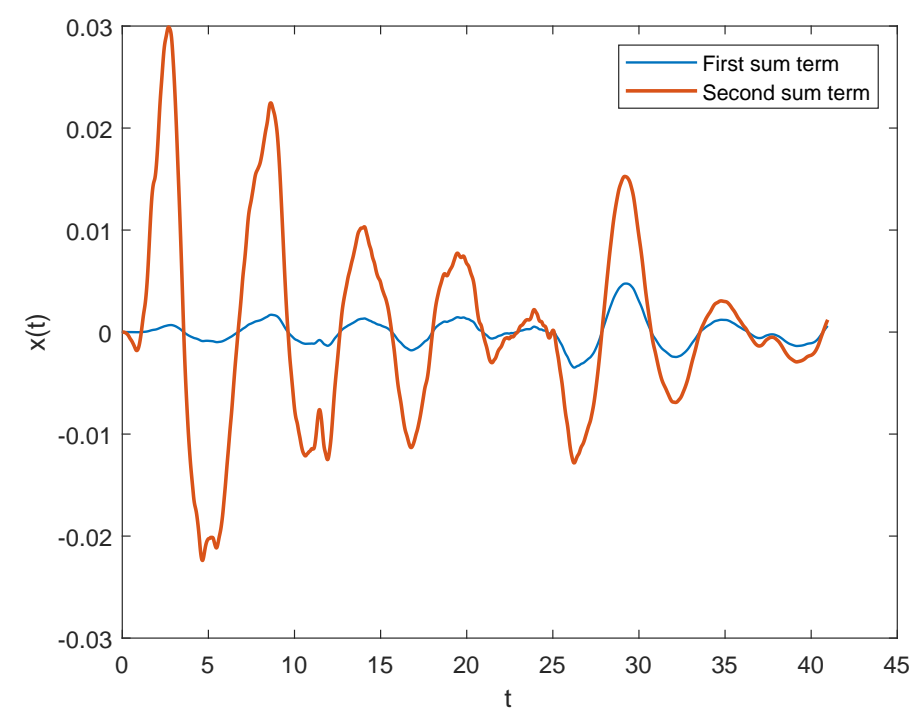

Fig. 17. Two response components of the SDOF fractional oscillator with $q_{1}=\pi / 30$ and $q_{2}=2 \pi / 7$ to the earthquake loading by the frequency domain method 

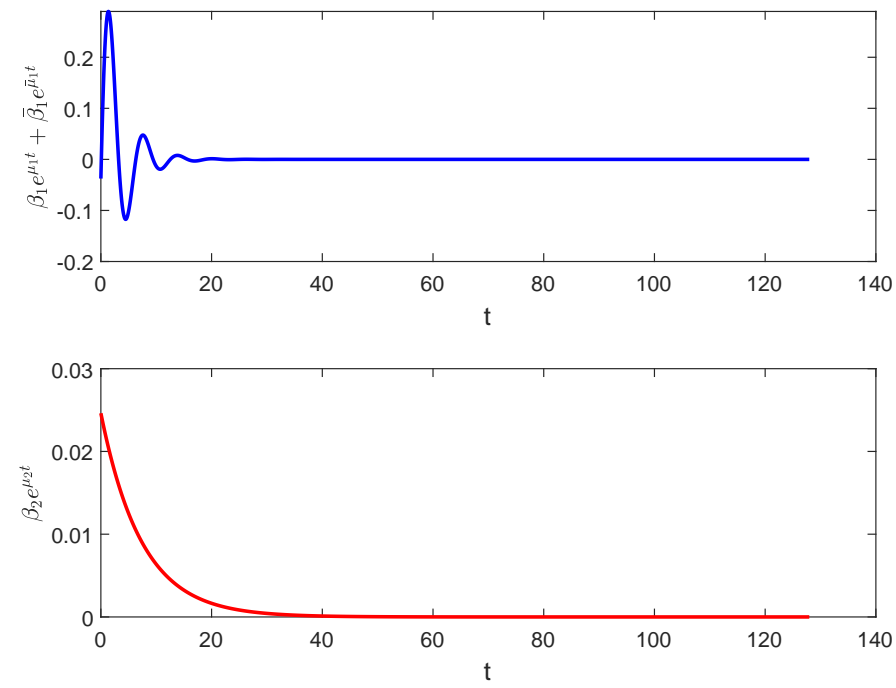

Fig. 18. Two components of the IRF for oscillator with $q_{1}=\pi / 30$ and $q_{2}=$ $2 \pi / 7$ : (a) dominated oscillatory term; (b) minor non-oscillatory term 

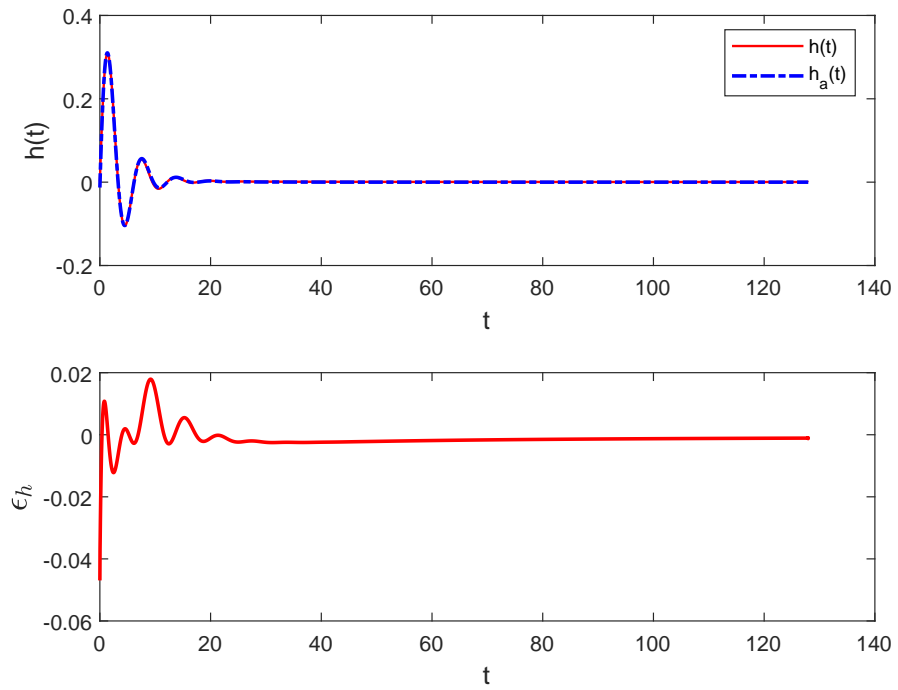

Fig. 19. The SDOF fractional oscillator with $q_{1}=\pi / 30$ and $q_{2}=2 \pi / 7$ : (a) Comparison between $h(t)$ and $h_{a}(t)$; (b) Relative error between $h(t)$ and $h_{a}(t)$ 


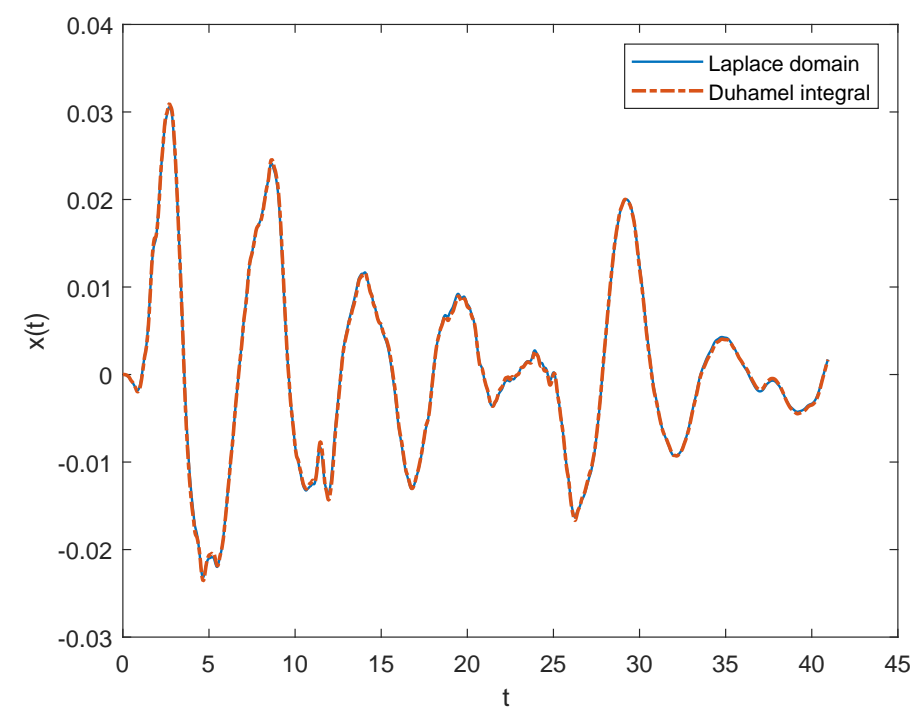

Fig. 20. Response of the SDOF fractional oscillator with $q_{1}=\pi / 30$ and $q_{2}=$ $2 \pi / 7$ to the earthquake loading based on the Laplace domain and Duhamel integral methods 